Case Study

\title{
Does kinematics add meaningful information to clinical assessment in post-stroke upper limb rehabilitation? A case report
}

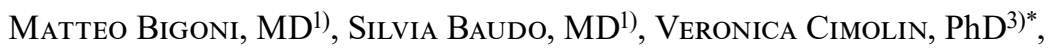 \\ Nicola Cau, PhD ${ }^{3)}$, Manuela Galli ${ }^{3,4)}$, Lucia Pianta, MD ${ }^{1)}$, Elena Tacchini, MD ${ }^{1,2}$, \\ Paolo Capodaglio, $\mathrm{MD}^{2)}$, Alessandro Mauro, $\mathrm{MD}^{1-5)}$ \\ 1) Department of Neurology and NeuroRehabilitation, Ospedale San Giuseppe, Istituto Auxologico \\ Italiano, IRCCS, Italy \\ 2) Orthopaedic Rehabilitation Unit and Clinical Lab for Gait Analysis and Posture, Ospedale San \\ Giuseppe, Istituto Auxologico Italiano, IRCCS, Italy \\ 3) Department of Electronics, Information and Bioengineering, Politecnico di Milano: P.zza Leonardo \\ da Vinci 32, 20133, Milano, Italy \\ 4) IRCCS "San Raffaele Pisana”, Tosinvest Sanità, Roma, Italy \\ 5) Department of Neuroscience "Rita Levi Montalcini", University of Torino, Italy
}

\begin{abstract}
Purpose] The aims of this case study were to: (a) quantify the impairment and activity restriction of the upper limb in a hemiparetic patient; (b) quantitatively evaluate rehabilitation program effectiveness; and (c) discuss whether more clinically meaningful information can be gained with the use of kinematic analysis in addition to clinical assessment. The rehabilitation program consisted of the combined use of different traditional physiotherapy techniques, occupational therapy sessions, and the so-called task-oriented approach. [Subject and Methods] Subject was a one hemiplegic patient. The patient was assessed at the beginning and after 1 month of daily rehabilitation using the Medical Research Council scale, Nine Hole Peg Test, Motor Evaluation Scale for Upper Extremity in Stroke Patients, and Hand Grip Dynamometer test as well as a kinematic analysis using an optoelectronic system. [Results] After treatment, significant improvements were evident in terms of total movement duration, movement completion velocity, and some smoothness parameters. [Conclusion] Our case report showed that the integration of clinical assessment with kinematic evaluation appears to be useful for quantitatively assessing performance changes. Key words: Stroke, Upper-limb kinematics, Rehabilitation
\end{abstract}

(This article was submitted Feb. 12, 2016, and was accepted May 7, 2016)

\section{INTRODUCTION}

Stroke is a leading cause of long-term disability in Western countries ${ }^{1)}$ : approximately $70-80 \%$ of stroke survivors have limited activities of daily living (ADL) due to motor impairment of the more affected upper limb. Many patients do not regain functional use of the paretic arm, and by 6 months post-stroke, a large proportion (25-50\%) remains dependent on others for at least one $\mathrm{ADL}^{2)}$. The effects of rehabilitative interventions are usually measured using clinical assessment tools and functional scales probing specific aspects of a subject's motor behavior. Existing clinical measures are widely accepted, standardized, and validated. However, these evaluations tend to be subjective and depend on the therapist who observes and rates the motor pattern using ordinal measurements scales. Other limits of clinical scales include low resolution and sensitivity ${ }^{3)}$ as well as the time required to perform them, all of which discourage their routine administration. Quantitative measurements

*Corresponding author. Veronica Cimolin (E-mail: veronica.cimolin@polimi.it)

(C2016 The Society of Physical Therapy Science. Published by IPEC Inc.

This is an open-access article distributed under the terms of the Creative Commons Attribution Non-Commercial No Derivatives (by-nc-nd) License $<$ http://creativecommons.org/licenses/by-nc-nd/4.0/>. 
of movement using movement analysis, on the other hand, convey a wealth of information about the quantity of motor behavior that is not evident from clinical evaluations. Instrumental analysis can be used to measure each segment and joint as well as kinematic parameters such as displacement, speed, and acceleration, but most importantly, it allows assessment of repeatability ${ }^{4,5)}$ and some particular feature such as movement smoothness ${ }^{6,7)}$. This is a very important advance in the evaluation of motor capacity because repeatability and smoothness are strictly linked to the patient's capacity for coordination and, consequently, selective motor control ${ }^{8}$. While for gait quantitative analysis of movement has reached almost universal standardization and represents an objective assessment used to measure clinical outcomes ${ }^{9,10)}$ in different pathologies in both children and adults, upper-limb kinematics is a much less frequently published topic, especially in stroke patients. Most of the studies on upper-limb kinematics are, in fact, on children with cerebral palsy ${ }^{11,12)}$, and to our knowledge, only a few studies in stroke patients ${ }^{2,3,7)}$ have been published to date.

Accordingly, and from the clinical need to quantitatively evaluate patients with stroke, the aims of this case study were to: (a) quantify the impairment and activity restriction of the upper limb in a hemiplegic patient after stroke using both clinicalfunctional scales and upper-limb kinematics; (b) quantitatively evaluate upper-limb rehabilitation program effectiveness over time; and (c) discuss whether more clinically meaningful information can be gained with the use of kinematic analysis in addition to clinical assessment compared to the latter alone.

\section{SUBJECT AND METHODS}

Our patient (M.A.) was a young (34-year-old) left-handed woman who presented with a stroke event due to occlusion of the left superior cerebellar artery. Acute symptoms included left upper-limb weakness, headache, vomiting, and dizziness. No significant diseases were previously reported, nor were significant vascular risk factors or cerebrovascular events evident. The clinical picture was characterized by mild left hemiparesis, mainly in the upper limb, reduced balance, and left-limb incoordination. Ultrasonography of the supra-aortic and intracranial arteries showed a hypoplastic right vertebral artery, while magnetic resonance imaging after 1 month documented a cerebellar left hemispheric and upper cerebellar vermis ischemic lesion. Instrumental examinations excluded cardiac embolism, and hematologic assessment was normal except for a low and nonspecific positivity for anti-nuclear antibodies. The onset of the patient's symptoms occurred about 2 months before the start of the rehabilitation program. At the first evaluation before the rehabilitative program, the patient was not able to use her left upper limb during ADL. The limb was perceived as a "passive weight", an "object" linked to the body during walking and active movements. All of the movements were severely limited by a strain-related tremor, especially during bilateral tasks.

The patient completed a 1-month rehabilitation program consisting of three 1-hour sessions a week. Motor training was based on the combined use of conventional physiotherapy techniques (mainly facilitation techniques), occupational therapy, and the so-called task-oriented approach ${ }^{13}{ }^{14}$. The latter is an approach based on the motor learning hypothesis in which each motor task is potentially related to an internal (cerebral) motor image, which is the underlying mechanism of imitation and learning processes (probably via the mirror neuron system $)^{15-17)}$. This technique was preceded by exercises based on motor imaging techniques (observation and imagination, according to Berends) ${ }^{18-20)}$ integrated with some exercises (i.e. drawing according to mirror therapy, drawing " 8 " number and " $\infty$ " symbol) based on the concept that new functional neuronal links are activated when an intentional movement is used ${ }^{21}$. Physiotherapy focused on exercises aimed at stabilizing the shoulder girdle considering the kinematics of reaching and the influence of the trunk, while occupational therapy included functional activities for the coordination of the upper limb's different muscle groups and the hand's fine motor skills. During these exercises, manual guidance was used as suggested by Carr and Shepard ${ }^{22}$ to wean the patient from direct assistance and foster independence.

The patient was assessed with the Medical Research Council ${ }^{23)}$ scale to test muscle strength, Nine Hole Peg Test ${ }^{24)}$ to test hand dexterity, Motor Evaluation Scale for Upper Extremity in Stroke Patients ${ }^{25}$ (MESUPES) to test residual functional upper-limb ability, and Hand Grip "Jamar" Dynamometer test ${ }^{26}$ ) to assess hand muscle strength. Further investigations used for the instrumental assessment included video recording and 3D movement analysis of the upper limb at pre-test during enrollment (before the intervention) and post-test at the end of the treatment ( 1 month after post-test). The instrumental test consisted of repetitive pointing movements, during which the participant was asked to reach a visual target. The task was considered representative of everyday functional activities, with similar tasks having been described in previous studies examining upper-limb movement ${ }^{2}$.

The subject was seated on a comfortable chair. A monitor placed on a table in front of her represented the target. The screen was placed at a distance from the acromion corresponding to $80 \%$ of the upper limb length (i.e. the distance between the acromion and the fingertip). In the starting position, the patient was asked to place her hands on the table surface directly in front of her with the elbow flexed at approximately $90^{\circ}$, the forearm slightly pronated, and the wrist in a neutral position. Neither the trunk nor the head was restrained. The reaching task involved leaning forward and extending the elbow to touch a stationary target (25-mm-diameter circle) positioned on the monitor. The subject was asked to touch the target as precisely as possible at a comfortable speed and then return her hand to the starting position. The task was completed separately by using the affected and unaffected arms. The task was repeated 36 times (three trials of six consecutive movements, left and right). To minimize the fatigue effect, she was allowed to rest $30 \mathrm{~s}$ between trials.

The kinematics of the upper limb were assessed using an optoelectronic system with passive markers (Vicon T40; Oxford 
Table 1. Patient's clinical assessment scores in the PRE, POST1, and POST2 sessions

\begin{tabular}{lccc}
\hline & PRE & POST1 & POST2 \\
\hline Strength MRC scale (left shoulder and elbow) & $3+/ 5$ & $4 / 5$ & $4 / 5$ \\
Strength MRC scale (left hand) & $4 / 5$ & $4+/ 5$ & $4+/ 5$ \\
Nine Hole Peg Test & 9 in 50” & 9 in 32” & 9 in $20 ”$ \\
MESUPES & $45 / 58$ & $54 / 58$ & $57 / 58$ \\
Hand grip dynamometer & mean $15.6 \mathrm{~kg}$ & mean $18 \mathrm{~kg}$ & mean $18 \mathrm{~kg}$ \\
\hline
\end{tabular}

PRE: before the treatment; POST1: after the last session; POST2: 6 months after the last session; MRC: Medical Research Council; MESUPES: Motor Evaluation Scale for Upper Extremity in Stroke Patients

Metrics Group, Oxford, UK) equipped with six cameras at a sampling rate of $100 \mathrm{~Hz}$ and synchronized with a video system. The passive markers were placed at special points of reference directly on the subject's skin ${ }^{1,27,28)}$. A total of 18 markers, each with a 15-mm diameter, were used to identify the position of the head, trunk, and upper limb (arm, forearm, and hand). The position and orientation of the upper limb were reconstructed using the following markers bilaterally: acromion, lateral epicondyle of the humerus, styloid processes of the ulna and radius, head of the second metacarpal bone, and fingernail of the index finger. The head position was reconstructed using four markers and the trunk position was estimated using the markers on the right and left acromion, spinous process of $\mathrm{C}$, and anterior trunk midline. Additional markers were placed on the screen and targets to create the 3D coordinates of the target plane. The 3D coordinates of each marker and their derivatives were computed using SmartAnalyzer software (BTS, Milan, Italy). The 3D coordinates of the markers were filtered using a low-pass second order zero-phase Butterworth filter (cut-off frequency $=10 \mathrm{~Hz}$ ), and the trajectory and velocity of the fingernail marker were computed. The biomechanical model, data filtering, and variable computation steps were implemented using SmartAnalyzer software (BTS).

Each movement was segmented into three sequential phases as described elsewhere ${ }^{29)}$ : going phase (i.e. toward the target); adjusting phase (i.e. dedicated to precisely locating the target); and returning phase (i.e. toward the initial position).

To evaluate the changes that occurred after the program, some parameters were identified and calculated in each session (pre-test and post-test); the analyzed parameters are as follows:

- Total Movement Duration (Total MD): total time required to complete each task; in addition, the durations of the previous three phases were computed (Going MD, MD of going phase; Adjusting MD, MD of adjusting phase; and Returning $\mathrm{MD}, \mathrm{MD}$ of returning phase);

- Mean Movement Velocity (MMV): computed during the going phase and represents the mean velocity of the fingernail marker;

- Adjusting Sway (AS): length of the 3D path described by the fingernail during the adjusting phase and a measure of the adjustments made to reach the final position that represents an expression of the degree of precision;

- Index of Curvature (IC): ratio of the fingernail 3D path length to the linear distance between the initial and final pointing positions that is representative of movement smoothness during the ongoing phase;

- Average Jerk (AJ): mean value of the derivative of the acceleration (i.e. Jerk) according to the following equation

$\mathrm{AJ}=\frac{1}{T} \int_{0}^{T}\left[\left(\frac{d^{3} x}{d t^{3}}\right)^{2}+\left(\frac{d^{3} y}{d t^{3}}\right)^{2}+\left(\frac{d^{3} z}{d t^{3}}\right)^{2}\right]^{1 / 2} d t$

where $x(t), y(t)$, and $z(t)$ are the $x, y$, and $z$ coordinates of the fingernail and $T$ was the movement duration. The AJ index decreases with increased movement smoothness and is often used as a measure of selective motor control quality ${ }^{30)}$.

- Number of Movement Units (NMU): number of velocity peaks that exceeded $10 \%$ of the peak velocity that aimed to capture the number of online corrections performed by subjects during the ongoing phase to avoid the inclusion of tremor components that occurs at the end of purposeful movements (when the velocity is low).

- Range of Motion at the elbow (ROM elbow) and shoulder (ROM shoulder): difference between the maximum and minimum values of the elbow and shoulder angles on the frontal plane (for shoulder joint) and sagittal plane (for shoulder and elbow joints) during the going phase, which were computed as described in previous studies ${ }^{29,31)}$.

While the head markers' trajectories were also acquired during this study, they are not included in the present analysis and not discussed herein. The patient provided written consent to voluntarily participate in accordance with the local ethical committee requirements. All of the previously defined parameters were computed for the participant for the affected and unaffected side in the two sessions and compared with the values of an age-matched control group (CG) that was assessed using the same experimental set-up and method ${ }^{1)}$. 
Table 2. Kinematic measures (mean and standard deviation) during reaching in the PRE, POST1, and POST2 sessions for the patient and the $\mathrm{CG}$

\begin{tabular}{|c|c|c|c|c|c|c|c|}
\hline & \multicolumn{2}{|c|}{ PRE session } & \multicolumn{2}{|c|}{ POST1 session } & \multicolumn{2}{|c|}{ POST2 session } & \multirow[t]{2}{*}{$\mathrm{CG}$} \\
\hline & Affected limb & $\begin{array}{l}\text { Unaffected } \\
\text { limb }\end{array}$ & Affected limb & $\begin{array}{l}\text { Unaffected } \\
\text { limb }\end{array}$ & Affected limb & $\begin{array}{l}\text { Unaffected } \\
\text { limb }\end{array}$ & \\
\hline \multicolumn{8}{|l|}{ MD } \\
\hline Total & $3.04(0.13)$ & $1.86(0.62)$ & $2.08(0.08)$ & $1.57(0.07)$ & $1.98(0.09)$ & $1.60(0.05)$ & $1.82(0.15)$ \\
\hline Going & $1.38(0.14)$ & $0.80(0.09)$ & $0.84(0.02)$ & $0.75(0.12)$ & $0.89(0.05)$ & $0.80(0.08)$ & $0.82(0.17)$ \\
\hline Adjusting & $0.53(0.18)$ & $0.23(0.03)$ & $0.24(0.07)$ & $0.16(0.04)$ & $0.22(0.10)$ & $0.29(0.05)$ & $0.28(0.15)$ \\
\hline Returning & $1.12(0.06)$ & $0.83(0.06)$ & $0.75(0.12)$ & $0.72(0.04)$ & $0.84(0.04)$ & $0.81(0.04)$ & $0.75(0.12)$ \\
\hline \multicolumn{8}{|l|}{ Velocity $(\mathrm{m} / \mathrm{s})$} \\
\hline MMV & $0.39(0.18)$ & $0.58(0.05)$ & $0.55(0.02)$ & $0.65(0.09)$ & $0.55(0.03)$ & $0.61(0.06)$ & $0.59(0.14)$ \\
\hline \multicolumn{8}{|c|}{ Movement Smoothness and Precision } \\
\hline IC & $1.43(0.12)$ & $1.10(0.03)$ & $1.23(0.03)$ & $1.11(0.02)$ & $1.22(0.07)$ & $1.12(0.03)$ & $1.09(0.15)$ \\
\hline $\mathrm{AJ}\left(\mathrm{mm} / \mathrm{s}^{3}\right)$ & $222.15(11.33)$ & $229.48(8.67)$ & $240.20(19.15)$ & $249.20(15.64)$ & $245.92(17.11)$ & $245.27(15.58)$ & $229.62(14.60)$ \\
\hline NMU & 15.20 & 2.4 & 5.20 & 2.61 & 5.60 & 3.2 & $2.77(1.45)$ \\
\hline $\begin{array}{l}\text { Frequency of direc- } \\
\text { tion changes }(\mathrm{Hz})\end{array}$ & 5.19 & 5.06 & 4.36 & 8.02 & 4.41 & 6.77 & $6.92(1.66)$ \\
\hline $\mathrm{AS}(\mathrm{mm})$ & 38.60 & 6.6 & 9.6 & 6.7 & 9.0 & 5.8 & $6.71(3.10)$ \\
\hline \multicolumn{8}{|l|}{ Angles $\left({ }^{\circ}\right)$} \\
\hline $\begin{array}{l}\text { ROM shoulder } \\
\text { flexion-extension }\end{array}$ & $36.45(3.49)$ & $34.91(0.81)$ & $31.50(1.71)$ & $43.8(1.98)$ & $36.73(2.10)$ & $45.32(1.98)$ & $37.91(3.40)$ \\
\hline $\begin{array}{l}\text { ROM shoulder } \\
\text { abduction-adduction }\end{array}$ & $13.88(3.81)$ & $11.06(2.54)$ & $12.22(0.70)$ & 10.54 (1.32) & $17.71(2.39)$ & $1.14(1.76)$ & $8.82(0.90)$ \\
\hline $\begin{array}{l}\text { ROM elbow } \\
\text { flexion-extension }\end{array}$ & $19.26(3.10)$ & $22.81(3.28)$ & $18.52(1.66)$ & $19.81(2.18)$ & $17.22(2.30)$ & $19.43(1.37)$ & $19.10(5.51)$ \\
\hline
\end{tabular}

PRE: before the treatment; POST1: after the last session; POST2: 6 months after the last session; CG: control group; MD: Movement Duration; MMV: Mean Movement Duration; IC: Index of Curvature; AJ: Average Jerk; AS: Adjusting Sway; NMU: Number of Movement Units; ROM: Range of Motion

\section{RESULTS}

The patient was able to complete the clinical and instrumental evaluation with 3D analysis. In the neurological examination, mild left hemiparesis but predominant left upper-limb ataxia were evident. The clinical scales used for the pre- and post-assessments are shown in Table 1.

The mean values (standard deviation) of all the kinematic parameters considered in this study for the pathological group in three sessions (PRE: before the treatment; POST1: after the last session; POST2: 6 months after the last session) and for the CG are shown in Table 2. The patient was able to complete the required task during each testing session.

In the PRE session, data obtained from the affected limb were different from those of the unaffected limb. The patient performed the movement more slowly with the affected arm (MMV and Total MD indices). The task execution duration of all of the phases (Going MD, Adjusting MD, and Returning MD indices) were longer than that in the unaffected limb and CG. The patient had higher IC values for the affected limb, with an AJ value close to the CG value.

While executing the movements, the subject showed a segmented velocity profile of the affected arm rather than a bellshaped profile as observed in normal subjects, and this feature was confirmed by the greater NMU value. The length of the $3 \mathrm{D}$ path described by the fingernail during the adjusting phase (AS index) was longer than the normality range, indicating lower precision performing the reaching movement as for the affected limb. The patient's ROM was similar to the CG value during the required task for the affected and unaffected arms.

After the treatment (POST1 session), no significant changes were observed for the unaffected arm during the reaching movement. Regarding movement duration of the affected upper limb, improvements were observed in Total MD, Going MD, Adjusting MD, Returning MD, and MMV. With regard to smoothness, IC improved and AJ slightly increased. The NMU index decreased significantly, reaching a value similar to that of the CG. The AS index decreased during movement, approaching normal values. No changes were evident in ROM of the upper-limb joints. From the functional point of view, soon after starting treatment, the patient reported greater overall use and spatial position awareness of the upper left limb during ADL. The results obtained in the POST1 session were maintained in the POST2 session, and no differences were observed between them. 


\section{DISCUSSION}

This case study aimed to provide evidence of the clinical usefulness of kinematic analysis in addition to clinical-functional scales in assessing outcomes in a stroke patient with upper-limb impairment undergoing rehabilitation. The clinical outcomes used in this case report were able to capture an improvement in hand dexterity and muscle strength (as indicated in particular by NHPT, Hand Grip, and MESUPES scores). An evident change in global scores of all the different clinical scales used documented the change in motor performance. However, clinical scores do not capture quantitative information due to the lack of specific indexes (i.e. smoothness and accuracy). In addition, intra- and inter-operator variability related to the use of such scales should always be considered a possible source of bias.

Here we evaluated a reaching movement with upper-limb kinematics. This task was selected among those proposed by the current literature because it represents a basic common ADL and is easy enough to be performed by all patients yet complex enough to highlight some specific upper-limb limitations after stroke.

At baseline, our patient performed the task more slowly and with less precision and smoothness compared to healthy subjects. After treatment, significant improvements were evident in terms of movement duration as demonstrated by the reduced total movement duration and increases in movement velocity at the POST1 and POST2 time points. Some positive changes were evident in terms of parameters directly connected to smoothness and precision. After treatment, the degree of adjusting sway improved during the requested task, thus demonstrating that the patient performed more accurate movements in the adjusting phase. The smoothness parameters improved after the rehabilitative period, especially for the IC index, which decreased after treatment to a mean value similar to that of the control group. It is important to highlight that the obtained improvements were maintained at the POST2 session.

Our data quantify post-stroke upper-limb impairment and positive rehabilitation outcomes using combined techniques. The kinematic analysis was capable of quantifying functional recovery after stroke. As for the latter aim of this study, the integration of clinical assessment and kinematic evaluation appears to be useful for quantitatively representing changes in performance, thus being a meaningful indicator of rehabilitation program effectiveness. This provides evidence of rehabilitation effectiveness for improving functional upper-limb movements after stroke and, ultimately, patient quality of life.

To our knowledge, no 3D upper limb kinematic studies have been conducted on stroke patients completing a similar treatment regimen. Combining the assessment of body function at the impairment level according to the International Classification of Functioning, Disability, and Health using 3D upper-limb kinematics and of activity level using clinical functional scales provides a comprehensive picture of the actual functional capacity of the paretic limb and its improvement potential.

One limitation of this study is that it evaluated only one predefined stereotyped movement. However, given the infinite combinations of movements that can be performed by the upper limb, it is necessary to define a priori which are the specific movements to be studied to obtain comparable data. A further limitation is that the model we used did not consider the hand.

In conclusion, this case report described a reliable and sensitive way to evaluate motor recovery and investigate the mechanism underlying functional improvement. We believe that supplementing the clinical-functional scales with objective outcome measures will aid the development of finer assessment tools for upper-limb function and outcome measures. Further studies will be needed to confirm these preliminary results on a large sample to allow us to better understand the mechanisms leading to upper-limb motor ability improvements after stroke.

\section{REFERENCES}

1) Menegoni F, Milano E, Trotti C, et al.: Quantitative evaluation of functional limitation of upper limb movements in subjects affected by ataxia. Eur J Neurol, 2009, 16: 232-239. [Medline] [CrossRef]

2) Caimmi M, Carda S, Giovanzana C, et al.: Using kinematic analysis to evaluate constraint-induced movement therapy in chronic stroke patients. Neurorehabil Neural Repair, 2008, 22: 31-39. [Medline] [CrossRef]

3) Balasubramanian S, Colombo R, Sterpi I, et al.: Robotic assessment of upper limb motor function after stroke. Am J Phys Med Rehabil, 2012, 91: S255-S269. [Medline] [CrossRef]

4) Jaspers E, Desloovere K, Bruyninckx H, et al.: Review of quantitative measurements of upper limb movements in hemiplegic cerebral palsy. Gait Posture, 2009, 30: 395-404. [Medline] [CrossRef]

5) Mackey AH, Walt SE, Stott NS: Deficits in upper-limb task performance in children with hemiplegic cerebral palsy as defined by 3-dimensional kinematics. Arch Phys Med Rehabil, 2006, 87: 207-215. [Medline] [CrossRef]

6) Schneiberg S, McKinley P, Gisel E, et al.: Reliability of kinematic measures of functional reaching in children with cerebral palsy. Dev Med Child Neurol, 2010, 52: e167-e173. [Medline] [CrossRef]

7) Marconi B, Filippi GM, Koch G, et al.: Long-term effects on cortical excitability and motor recovery induced by repeated muscle vibration in chronic stroke patients. Neurorehabil Neural Repair, 2011, 25: 48-60. [Medline] [CrossRef]

8) Don R, Capodaglio P, Cimolin V, et al.: Instrumental measures of spinal function: is it worth? A state-of-the art from a clinical perspective. Eur J Phys Rehabil Med, 2012, 48: 255-273. [Medline]

9) Gage JR: The treatment of gait problems in cerebral palsy. Gage JR (ed.) London: Cambridge University Press, 2004.

10) Lee EH, Goh JC, Bose K: Value of gait analysis in the assessment of surgery in cerebral palsy. Arch Phys Med Rehabil, 1992, 73: 642-646. [Medline] 
11) Butler EE, Ladd AL, Louie SA, et al.: Three-dimensional kinematics of the upper limb during a Reach and Grasp Cycle for children. Gait Posture, 2010, 32: 72-77. [Medline] [CrossRef]

12) Coluccini M, Maini ES, Martelloni C, et al.: Kinematic characterization of functional reach to grasp in normal and in motor disabled children. Gait Posture, 2007, 25: 493-501. [Medline] [CrossRef]

13) Bernstein N: The coordination and regulation of movements. Oxford: Pergamon Press, 1967.

14) Nelles G, Jentzen W, Jueptner M, et al.: Arm training induced brain plasticity in stroke studied with serial positron emission tomography. Neuroimage, 2001, 13: 1146-1154. [Medline] [CrossRef]

15) Page SJ, Levine P, Sisto S, et al.: A randomized efficacy and feasibility study of imagery in acute stroke. Clin Rehabil, 2001, 15: 233-240. [Medline] [CrossRef]

16) Jackson PL, Lafleur MF, Malouin F, et al.: Potential role of mental practice using motor imagery in neurologic rehabilitation. Arch Phys Med Rehabil, 2001, 82: 1133-1141. [Medline] [CrossRef]

17) Johnson-Frey SH: Stimulation through simulation? Motor imagery and functional reorganization in hemiplegic stroke patients. Brain Cogn, 2004, 55: 328-331. [Medline] [CrossRef]

18) Cicinelli P, Marconi B, Zaccagnini M, et al.: Imagery-induced cortical excitability changes in stroke: a transcranial magnetic stimulation study. Cereb Cortex, 2006, 16: 247-253. [Medline] [CrossRef]

19) Gallese V, Fadiga L, Fogassi L, et al.: Action recognition in the premotor cortex. Brain, 1996, 119: 593-609. [Medline] [CrossRef]

20) Berends HI, Wolkorte R, Ijzerman MJ, et al.: Differential cortical activation during observation and observation-and-imagination. Exp Brain Res, 2013, 229: 337-345. [Medline] [CrossRef]

21) Dennison P: Personalized Whole Brain Integration: the basic II manual on Educational Kinesiology. Edukinesthetics, 1985.

22) Carr JH, Shepherd RB: A motor learning model for rehabilitation. In: Carr JH, Shepherd RB (eds.), Movement Science. Foundations for Physical Therapy in Rehabilitation, 2nd ed. Gaithersburg: Aspen Publishers, 2000, pp 33-110.

23) Medical Research Council: Aids to the examination of the peripheral nervous system. Memorandum n45. London 1976.

24) Croarkin E, Danoff J, Barnes C: Evidence-based rating of upper-extremity motor function tests used for people following a stroke. Phys Ther, 2004, 84: 62-74. [Medline]

25) Van de Winckel A, Feys H, van der Knaap S, et al.: Can quality of movement be measured? Rasch analysis and inter-rater reliability of the Motor Evaluation Scale for Upper Extremity in Stroke Patients (MESUPES). Clin Rehabil, 2006, 20: 871-884. [Medline]

26) Hanten WP, Chen WY, Austin AA, et al.: Maximum grip strength in normal subjects from 20 to 64 years of age. J Hand Ther, 1999, 12: 193-200. [Medline] [CrossRef]

27) Molteni E, Cimolin V, Preatoni E, et al.: Towards a biomarker of motor adaptation: integration of kinematic and neural factors. IEEE Trans Neural Syst Rehabil Eng, 2012, 20: 258-267. [Medline] [CrossRef]

28) Rigoldi C, Molteni E, Rozbaczylo C, et al.: Movement analysis and EEG recordings in children with hemiplegic cerebral palsy. Exp Brain Res, 2012, 223: 517-524. [Medline] [CrossRef]

29) Cimolin V, Beretta E, Piccinini L, et al.: Constraint-induced movement therapy for children with hemiplegia after traumatic brain injury: a quantitative study. J Head Trauma Rehabil, 2012, 27: 177-187. [Medline] [CrossRef]

30) Feng CJ, Mak AF: Three-dimensional motion analysis of the voluntary elbow movement in subjects with spasticity. IEEE Trans Rehabil Eng, 1997, 5: 253-262. [Medline] [CrossRef]

31) Rab G, Petuskey K, Bagley A: A method for determination of upper extremity kinematics. Gait Posture, 2002, 15: 113-119. [Medline] [CrossRef] 